\title{
Comparison of the Effects of Electroacupuncture and Melatonin on Nerve Regeneration in Experimentally Nerve-Damaged Rats
}

\author{
Yasemin Özkan ${ }^{1, *}$, Mehmet Turgut ${ }^{2}$, Yasemin Turan', Mehmet Dinçer Bilgin ${ }^{3}$, Sinem Sari", \\ Mustafa Yilmaz ${ }^{3}$, Yiğit Uyanikgil ${ }^{5}$, Mahmut Alp Kiliç³, Derya Tanriöver ${ }^{5}$, Zehra Seznur Kasar ${ }^{1}$ \\ ${ }^{1}$ Department of Physical Therapy and Rehabilitation, Faculty of Medicine, Adnan Menderes University, Aydin, Turkey \\ ${ }^{2}$ Department of Neurosurgery, Faculty of Medicine, Adnan Menderes University, Aydin, Turkey \\ ${ }^{3}$ Department of Biophysics, Faculty of Medicine, Adnan Menderes University, Aydin, Turkey \\ ${ }^{4}$ Department of Anesthesia, Faculty of Medicine, Adnan Menderes University, Aydin, Turkey \\ ${ }^{5}$ Department of Histology-Embryology, Ege University, Izmir, Turkey
}

Received December 2, 2020

Revised June 22, 2021

Accepted August 17, 2021

Correspondence to

Yasemin Özkan

Department of Physical Therapy and

Rehabilitation, Faculty of Medicine,

Adnan Menderes University, Aydin,

Turkey

E-mail ftrjo@hotmail.com
Background: Development of methods to accelerate nerve regeneration in peripheral nerve damage is important. Electroacupuncture is a new therapeutic method that combines traditional acupuncture with modern electrotherapy. Melatonin has been shown to reduce nerve damage.

Objectives: In this study, we aimed to determine and compare the therapeutic effects of electroacupuncture and melatonin on rat sciatic nerve injury.

Methods: A total of 56 adult male Wistar Albino rats were divided into four study groups with 14 animals in each group: intact control (group I), subcutaneous saline (group II), subcutaneous melatonin (group III), and electroacupuncture (group IV). Surgical procedure including unilateral (right) sciatic nerve injury was applied to groups II, III, and IV. Saline and melatonin started immediately after surgery for six weeks, while electroacupuncture was given two weeks after surgery for 3 weeks. Functional and histological assessments were used as outcome measurements.

Results: Sciatic nerve damage caused a significant decrease in nerve conduction velocity. Both electroacupuncture treatment and melatonin treatment significantly increased the nerve conduction velocity. Both sciatic functional recovery and histological regeneration were faster in these treatment groups compared to the saline. However, no significant difference was observed between the two treatment groups.

Conclusion: Electroacupuncture and melatonin are promising alternative treatment strategies for peripheral nerve damage and can be examined in detail in future studies.

Keywords: Sciatic nerve injury, Electro-acupuncture, Melatonin, Sciatic function index, Nerve conduction velocity

\section{INTRODUCTION}

Today, peripheral nerve injuries are very common. They can occur in all kinds of accidents, especially traffic and work accidents, due to reasons such as piercing, nerve crush, cut and strain caused by cutting, stinging and firearms. Significant histopathological changes occur in the distal and proximal parts of the nerve following injury. Whatever the cause of the injury, the degenerative changes that occur after nerve damage are the same [1-3]. The success of nerve repairs in peripheral nerve injuries has increased significantly with the widespread use of microsurgical methods and the development of histological methods [4]. Nerve cells and neuroglia are responsible for the response that occurs after nerve tissue injury. Since the nerve cells are postmitotic cells, they cannot be regenerated as a result of severe injuries and death. However, nerve cells may respond as degeneration or regeneration, depending on the degree of injury to the axon [5].

Melatonin, an important secretion product of the pineal gland, is a powerful antioxidant that can easily pass through cell membranes and the blood-brain barrier. Melatonin's 
stimulating effect on glutathione synthesis, an important intracellular antioxidant, its effectiveness in reducing electron leakage from the mitochondrial electron transport system, and its synergistic effect with other antioxidants are held responsible for the mechanism of its antioxidant property [6]. Studies show that melatonin is both a direct free radical catcher and an indirect antioxidant [7].

Electroacupuncture is a new therapy method that combines traditional acupuncture with modern electrotherapy. With advances in modern science and technology, infrared, ultraviolet and laser rays, electromagnetic field and electrostimulation have entered the field of acupuncture. Among them, electrostimulation is considered the most important step. The fact that electroacupuncture is technically controllable, reproducible and easy to apply reduces the need for manual needle stimulation [8]. Although there are data on the effectiveness of acupuncture in traumatic brain injury, spinal cord injury, cerebral palsy and transverse myelitis, more longterm and controlled studies are required [9].

The sciatic nerve model in rats is widely used to examine functional changes after nerve injury and to investigate the effectiveness of different surgical methods and medical treatments. Experimental studies have shown that rat sciatic nerve injury results in lipid peroxidation and causes free radical production that damages the nerve tissue.

Until now, the effects of both melatonin and electroacupuncture on peripheral nerve injury have been evaluated in a limited number of studies. However, no comparative study examining the effects of these two methods on the peripheral nervous system was found in the literature review. Therefore, in this experimental study, it is aimed to investigate the comparative effects of electroacupuncture and melatonin on damaged nerve tissue.

\section{MATERIALS AND METHODS}

This experimental study was conducted in Aydın Adnan Menderes University Physical Medicine and rehabilitationDepartment Laboratory. This study was approved by Adnan Menderes University Animal Experiments Local Ethics Committee (ADÜ HADYEK) (Ethics committee approval number: TPF-18006).

\section{Study groups}

A total of 56 adult male Wistar Albino rats weighing 250300 grams were used for this study. The rats were divided into four study groups with 14 animals in each cage: intact control (group I), subcutaneous saline (group II), subcutaneous melatonin (group III), and electroacupuncture (group IV). Surgical procedure including unilateral (right) sciatic nerve injury was applied to groups II, III, and IV.

\section{Electroacupuncture}

In the electroacupuncture procedure, 3 stainless steel acupuncture needles with a diameter of $0.3 \mathrm{~mm}$ connected to the exit terminals of the stimulation device were inserted at a depth of 2-3 $\mathrm{mm}$ at acupuncture points. Two different stimuli were performed simultaneously, a dense-disperse waves of $1-20 \mathrm{~Hz}$ was applied for 30 minutes at points along the path of the sciatic nerve and at point ST36, and a pulse stimulus with $70 \mathrm{~Hz}$ waves was applied for 15 minutes at points GB30 and GB34. In order to provide various positive effects of acupuncture such as improving neuropathic pain, motor functions, and neuroplasticity, and inhibiting anxiety in rats, the points encompassing GB30, GB34, and ST36 were used for stimulation with electroacupuncture [10-12]. The current intensity was slightly below the level that induced the apparent muscle contraction. Electroacupuncture procedure was began two weeks after surgery and performed with 72 hours intervals, for three weeks, and thus in seven sessions in total [13].

\section{Melatonin application}

Melatonin administration to the determined group in the study was determined as daily subcutaneous injection at a dose of 30 micrograms/100 g body weight. Melatonin and saline applications started immediately after surgery for six weeks.

\section{Surgical procedure}

Surgically, after a longitudinal incision in the right thigh of each rat under sterile conditions and anesthesia, compression and ischemia were created in the sciatic nerve by an "aneurysmclip" for 5 minutes. After this process, the wound was left to heal by covering the wound layer by layer.

\section{Measurement tools}

Sciatic functional index (SFI) and nerve conduction velocity were measured six weeks after surgical procedure. SFI values were calculated by performing "gait analysis" for each subject and measured once a week for four weeks.

Subsequently, all animals were sacrificed by cervical dislocation euthanasia. At the end of the experiment, right sciatic nerve specimen samples were obtained from animals in each group; half $(n=7)$ randomly selected for histological examination by microscopy.

Histopathological changes (regeneration findings) and damage to myelin sheaths and axons in the surgical specimens of animals treated with treatments mentioned were compared histologically.

\section{Histological evaluation}

Edema and damage in myelin sheaths and axons in tissue 
samples separated during surgery were evaluated. For this, fresh samples were taken for 24-48 hours in a mixture of 0 , M Cacodylate buffered $2 \%$ paraformaldehyde- $2 \%$ glutaraldehyde. Then, after postfixation with osmic acid, routine electron microscopic monitoring was performed and the samples were blocked in epon resin. 1 micron thin sections were taken with an ultratome and routine histopathological evaluation was performed with toluidine blue. After epon removal with $\mathrm{NaOH}$-Ethanol and sodium periodate, Hematoxylin\&Eosin and immunohistochemically staining the S-100 intermediate filament in the schwann cell cytoplasm was applied to anti-S100 and TUNEL staining procedures were applied to stain the apoptotic cells. S100 positive cell counts in 10 independent fields in 5 different preparations of each group were counted by two blind histologists.

\section{Statistical analysis}

Data belonging to each group are overwritten with Microsoft Office Excel 2010 version and IBM SPSS Statistics ver. 25.00 (Statistical Package for Social Sciences) program and statistical analyzes were performed with the same program. Statistical significance between the groups was analyzed using the One-Way ANOVA test with Tukey's test as post-hoc analysis and the Kruskal-Wallis test with Mann-Whitney U test as post-hoc analysis.

\section{RESULTS}

\section{Function recovery results}

Sciatic nerve damage caused a significant decrease in nerve conduction velocity. On the other hand, both electroacupuncture treatment and melatonin treatment caused a significant increase in nerve conduction velocity. According to the results of sciatic functional index, sciatic function recovery was faster in these two treatment groups compared to the Salinegroup. However, no significant difference was observed between the two treatment groups (Table 1).

\section{Histological regeneration results}

In the intact control group, when the sciatic nerve samples were examined, it was seen that the outer epineurium, the perineurium surrounding the nerve fascicules and the endoneurium connective tissue sheaths surrounding each axon were found to have normal histological structure. When axonal structures were evaluated, it was found that myelinated (large, medium, small) axons and unmyelinated axons and Schwann cell bands were in normal histological figuration. In addition, the distribution of small, medium and large myelinated axons also has a normal histological appearance. Generally smooth axons were detected with sections passing through Schwann cell nuclei in small diameter myelinated axons. It was also seen that the vein sections passing through the section plane were in normal structure. S-100 staining of the preparations in this group showed significant staining in Schmidt-Lantermanclefts and large axons in paranodal spaces (Fig. 1).

In the saline group, thickening of the connective tissue sheaths of the epineurium and perineurium was detected. Significant edema was detected in large diameter myelinated axons. When evaluated in terms of the distribution of small, medium and large myelinated axons, it was observed that small myelinated axons were located densely in places. In addition to degeneration and deletion in myelin rings, leakage and structural degeneration in axoplasmic areas were observed. It is observed that axon and myelin sheath structures are degenerated in a significant part of myelinated nerve fibers. The presence of macrophage and mast cells between the nerve fibers was detected. It was seen that these cells, which are evidence of the presence of inflammation, were found in large numbers around degenerate structures. It was observed that there was a thickening of the vessel barriers passing through the section plane. In terms of endothelial cells, it was found that they were in a relatively flat form compared to the control group and that they were deleted from time to time. When the vessel lumen was examined, there was a sign of stenosis. In this section it overlaps with the

Table 1. Nerve conduction velocity (NCV) and sciatic functional index (SFI) results after sciatic nerve damage

\begin{tabular}{lcccc}
\hline & Intact control & Saline & Melatonin & Electroacupuncture \\
\hline $\begin{array}{l}\text { NCVsix weeks after damage } \\
\text { SFI (after damage) }\end{array}$ & $76.84 \pm 3.89$ & $19.79 \pm 1.55^{*}$ & $27.06 \pm 2.39^{*, *}$ & $28.82 \pm 2.14^{*, *}$ \\
$\quad$ & & & \\
$6^{\text {th }}$ week & $-9.92 \pm 2.42$ & $-62.21 \pm 9.15^{*}$ & $-52.73 \pm 8.14^{*, *}$ & $-52.24 \pm 7.25^{*, *}$ \\
$7^{\text {th }}$ week & $-10.35 \pm 3.29$ & $-35.07 \pm 8.92^{*}$ & $-25.20 \pm 8.04^{*, *}$ & $-29.45 \pm 3.88^{*}$ \\
$8^{\text {th }}$ week & $-7.33 \pm 1.53$ & $-21.85 \pm 6.01^{*}$ & $-12.79 \pm 3.81^{*, *}$ & $-13.42 \pm 2.82^{*, *}$ \\
$9^{\text {th }}$ week & $-12.64 \pm 2.57$ & $-14.86 \pm 3.67$ & $-9.68 \pm 1.38^{*}$ & $-11.99 \pm 2.65^{\ddagger}$ \\
\hline
\end{tabular}

Statistical significance between the groups was analyzed using the One-Way ANOVA test with Tukey's test as post-hoc analysis. ${ }^{*} p<0.001$, when compared to intact control group; ${ }^{\sharp} p<0.01$, when compared to the Saline group; ${ }^{*} p<0.05$, when compared to the Saline group. 

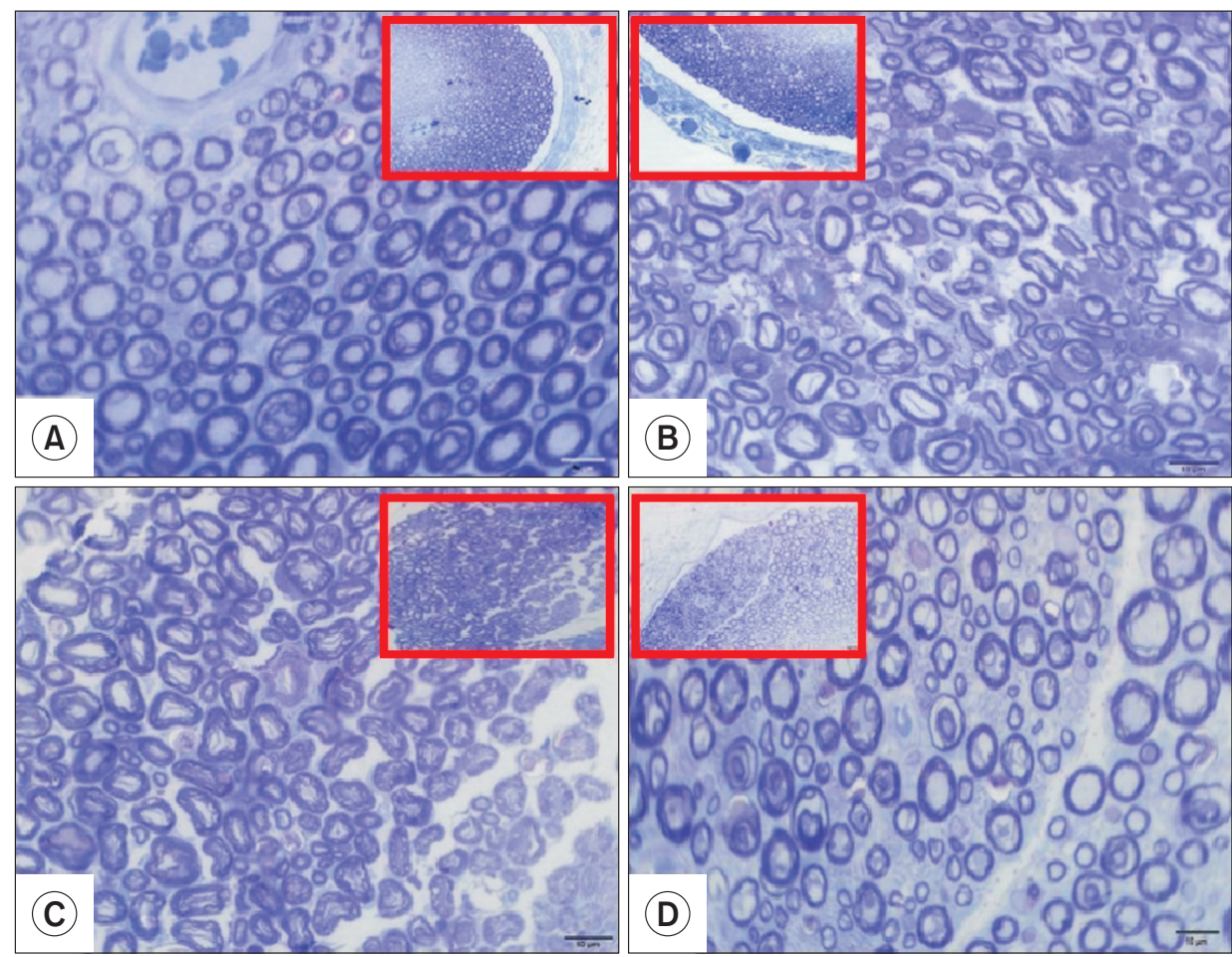

Fig. 1. Histological appearance of myelinated axons. Sciatic nerve structure. $\times 100$ magnification (thumbnails $\times 4$ magnification). Toluidine Blue Staining. (A) Intact control group. (B) Saline group. (C) Melatonin group. (D) Electroacupuncture group.

existence of a general edematous condition. S-100 staining shows significant positive reactivity inSchmidt-Lanterman clefts of large diameter axons. However, scattered and faint positive immunreactivity was detected among the lamellae of large-diameter myelinated fibersseparated myelin rings.

In the melatonin group, it was observed that the epineurium and perineurium structure was similar to the control group. In the large-diameter myelinated axons observed in the surgical group, it was found that prominent edema continued in places. When evaluated in terms of the distribution of small, medium and large myelinated axons, it was seen that the axon distribution was between the control and saline groups. When compared with the saline group, minimal degeneration anddiscontinuity were observed in myelin rings. Leakageand structural degeneration were found to be minimal in axoplasmic areas. It was found that the thickening of the walls of the veins passing through the section plane reversed and was close to the control group (Fig. 2).

In terms of endothelial cells, the presence of cells in a configuration close to the control group was detected. It was observed that there was no condition associated with stenosis and stenosis in the vessel lumen. S-100 staining of the preparations in this group showed significant staining in Schmidh-Lanterman clefts and large axons in paranodal spaces. The number of stained cells in the melatonin group was fewer than that of the intact control group, but more than that of saline group $(p<0.001)$.

In the electroacupuncture group, it was observed that the epineurium and perineurium structure were similar to the control groupbut minimal edema was detected in large-diameter myelinated axonslike observed in the saline group. When evaluated in terms of the distribution of small, medium and large myelinated axons, it was seen that the axon distribution was between the control and melatonin groups. When compared with the saline group, minimal degeneration anddiscontinuity were observed in the myelin rings. Leakageand structural degeneration were found to be minimal in axoplasmic areas. It was observed that especially large-diameter axons had a similar structure to the control group, and medium and small-diameter axons were numerically smaller than the other groups. The presence of macrophage and mast cells between nerve fibers was detected in this group as well as in the saline group. Unlike the saline group, it was observed that the number of macrophages and mast cells was higher. It was determined that the thickening of the walls of the vessels passing through the section plane was at a low level and gave an appearance between the control, melatonin and saline groups. In terms of endothelial cells, it was found that the melatonin group was confronted with the saline group. Minimal stenosis and stenosis weredetected in the vessel lumen. S-100 staining of the preparations in this group showed significant staining in Schmidh-Lanterman clefts and large axons in paranodal spaces. It was determined that the number of stained cells was less than the control group and more than the saline group $(p<0.001)$ (Fig. 3). 

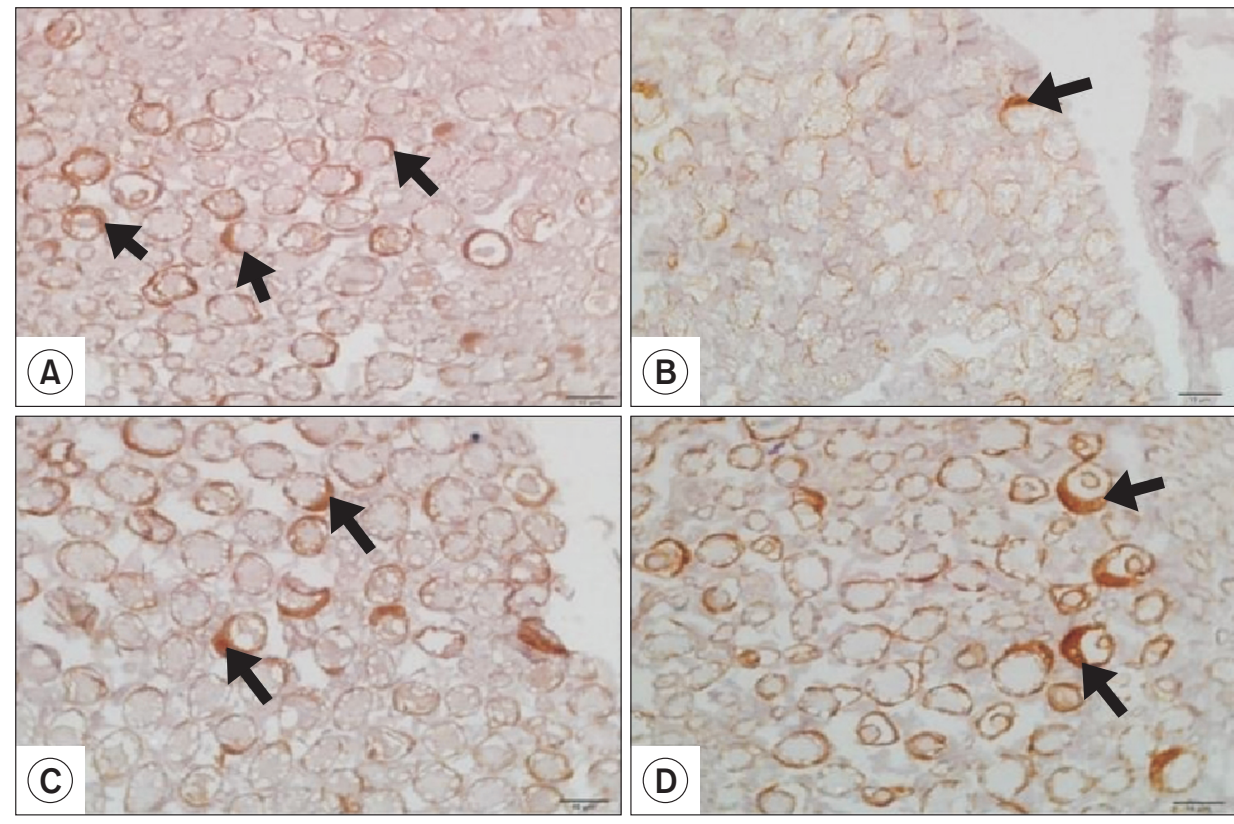

Fig. 2. Appearance of histological regeneration. Anti-S100 Staining. Black arrow: S-100 positive cells. $\times 100$ magnification. (A) Intact control group. (B) Saline group. (C) Melatonin group. (D) Electroacupuncture group.

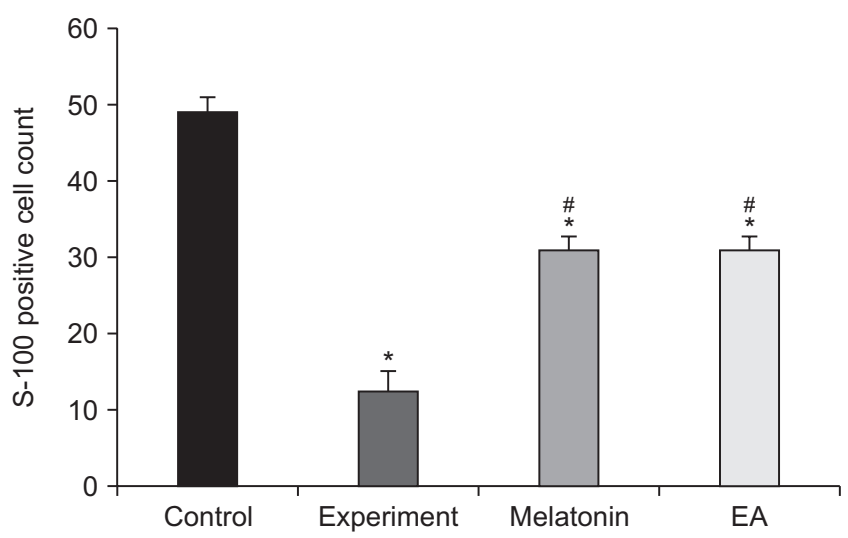

Fig. 3. Comparisons between the groups in terms of the anti-S100 positive cell count (the number of stained cells). Statistical significance between groups was analysed by Kruskal Wallis test with Mann Whitney $U$ test. ${ }^{*} p<0.001$, when compared to intact control group, $" p<0.001$, when compared to Saline group. EA = Electroacupuncture group.

\section{DISCUSSION}

In this study, the comparative effects of electroacupuncture, melatonin and saline were investigated. As result, both electroacupuncture and melatonin caused faster functional recovery and histological regeneration compared to the saline. However, no significant difference was observed between the two treatment groups.

It has been reported in the literature that low frequency electroacupuncture and acupuncture can improve sciatic nerve regeneration [14-18]. The in vitro nerve growth promoting effects of electrical stimulation have been demon- strated by in vivo experiments demonstrating that nerve regeneration can be increased by applying direct current to the sciatic nerve of rats while placing the cathode towards the distal end of the injured nerve canals [19]. Studies have revealed that acupuncture and electroacupuncture increase regenerated nerve Schwann cell proliferation and provide blood for treatments [20]. Some researchers have pointed out that when the nerve is processed, electrical stimulation will help restructure blood through the somatic/autonomic reflex arc to ensure adequate blood flow for the regenerated nerve [21].

In our study, it was aimed to determine and compare the therapeutic effects of electroacupuncture and melatonin on rat sciatic nerve injury. Although there are numerous studies detailing the effects of melatonin in various tissues, only a limited number of studies have documented its effects on peripheral nerves. In a study, it was observed that SOD activity was increased in the group treated with melatonin. It can be used to accelerate neural recovery and treat nerve damage, and has been observed to result in better nerve regeneration, especially when treated in dark mice [22]. In our study, a slight increase was observed in the melatonin applied groups. Similarly, another study reported that melatonin treatment increased SOD activity in sciatic nerve samples in rats after both cut and crush injuries [23].

If peripheral nerve regeneration can be accelerated in some way, much better functional recovery can be achieved if regenerated axons can be directed to restore their old connections in the periphery faster. It can be achieved with electroacupuncture. Recently, the application of electroacupuncture to stimulate nerve regeneration has become 
the main therapy in clinical rehabilitation and related basic research. Although we applied two different treatment groups in our study, no significant difference was observed between those. However, electroacupuncture studies are promising for the future.

\section{CONCLUSIONS}

This study provides data showing electroacupuncture and melatonin have potential effects on the regeneration of sciatic nerves in terms of functional recovery and histological regeneration. Peripheral nerve injuries are among the important clinical issues, and research is ongoing for further information on their treatment. Electroacupuncture is a promising alternative treatment strategy in the treatment of peripheral nerve damage and can be studied in detail in future studies. Melatonin is considered to have beneficial effects on axon length and sprouting following damage to peripheral nerves [24]. However, some studies show the disadvantageous effects of melatonin on peripheral nerves [25]. Therefore, further clinical and experimental studies should be conducted to shed light on the beneficial or harmful effects of different doses of melatonin treatment.

\section{DATA AVAILABILITY}

The corresponding author can be contacted to access the interested data about the study.

\section{CONFLICT OF INTEREST}

The authors declare no conflict of interest.

\section{ORCID}

Yasemin Özkan, https://orcid.org/0000-0003-3797-483X Mehmet Turgut, https://orcid.org/0000-0001-7130-2530

Yasemin Turan, https://orcid.org/0000-0002-4650-0567 Mehmet Dinçer Bilgin,

https://orcid.org/0000-0003-0754-0854

Yiğit Uyanikgil, https://orcid.org/0000-0002-4016-0522

Mahmut Alp Kiliç, https://orcid.org/0000-0003-2645-1988

Zehra Seznur Kasar,

https://orcid.org/0000-0001-9226-0659

\section{REFERENCES}

1. Robinson LR. Traumatic injury to peripheral nerves. Muscle Nerve 2000;23:863-73.

2. Liuzzi FJ, Tedeschi B. Peripheral nerve regeneration. Neurosurg Clin N Am 1991;2:31-42.
3. Burnett MG, Zager EL. Pathophysiology of peripheral nerve injury: a brief review. Neurosurg Focus 2004;16:E1.

4. Frostick SP, Yin Q, Kemp GJ. Schwann cells, neurotrophic factors, and peripheral nerve regeneration. Microsurgery 1998; 18:397-405.

5. Uyanikgil Y, Cavusoglu T, Kılıc KD, Yigitturk G, Celik S, Tubbs RS, et al. Useful effects of melatonin in peripheral nerve injury and development of the nervous system. J Brachial Plex Peripher Nerve Inj 2017;12:e1-6.

6. León J, Escames G, Rodríguez MI, López LC, Tapias V, Entrena A, et al. Inhibition of neuronal nitric oxide synthase activity by N1-acetyl-5-methoxykynuramine, a brain metabolite of melatonin. J Neurochem 2006;98:2023-33.

7. Reiter RJ, Guerrero JM, Escames G, Pappolla MA, AcuñaCastroviejo D. Prophylactic actions of melatonin in oxidative neurotoxicity. Ann N Y Acad Sci 1997;825:70-8.

8. Ulett GA, Han S, Han JS. Electroacupuncture: mechanisms and clinical application. Biol Psychiatry 1998;44:129-38.

9. Tekeoğlu İ, Tekeoğlu HN. [Acupuncture in the rehabilitation clinics]. J Biotechnol Strateg Health Res 2019;3(Özel Sayı):131-7. Turkish.

10. Zhang CH, Ma ZZ, Huo BB, Lu YC, Wu JJ, Hua XY, et al. Diffusional plasticity induced by electroacupuncture intervention in rat model of peripheral nerve injury. J Clin Neurosci 2019;69:250-6.

11. Park HJ, Kim HY, Hahm DH, Lee H, Kim KS, Shim I. Electroacupuncture to ST36 ameliorates behavioral and biochemical responses to restraint stress in rats. Neurol Res 2010;32 Suppl 1:111-5.

12. Jang JH, Yeom MJ, Ahn S, Oh JY, Ji S, Kim TH, et al. Acupuncture inhibits neuroinflammation and gut microbial dysbiosis in a mouse model of Parkinson's disease. Brain Behav Immun 2020;89:641-55.

13. Kalender AM, Dogan A, Bakan V, Yildiz H, Gokalp MA, Kalender M. Effect of Zofenopril on regeneration of sciatic nerve crush injury in a rat model. J Brachial Plex Peripher Nerve Inj 2009;4:6.

14. Yao CH, Chang RL, Chang SL, Tsai CC, Tsai FJ, Chen YS. Electrical stimulation improves peripheral nerve regeneration in streptozotocin-induced diabetic rats. J Trauma Acute Care Surg 2012;72:199-205.

15. Bertelli JA, Mira JC. Behavioral evaluating methods in the objective clinical assessment of motor function after experimental brachial plexus reconstruction in the rat. J Neurosci Methods 1993;46:203-8.

16. Chen YS, Yao CH, Chen TH, Lin JG, Hsieh CL, Lin CC, et al. Effect of acupuncture stimulation on peripheral nerve regeneration using silicone rubber chambers. Am J Chin Med 2001;29:377-85.

17. Huang J, Lu L, Zhang J, Hu X, Zhang Y, Liang W, et al. Electrical stimulation to conductive scaffold promotes axonal 
regeneration and remyelination in a rat model of large nerve defect. PLoS One 2012;7:e39526.

18. Liang F, Chen R, Cooper EL. Neuroendocrine mechanisms of acupuncture. Evid Based Complement Alternat Med 2012;2012: 792793.

19. Yannas IV, Zhang M, Spilker MH. Standardized criterion to analyze and directly compare various materials and models for peripheral nerve regeneration. J Biomater Sci Polym Ed 2007;18:943-66.

20. Chen YS, Hu CL, Hsieh CL, Lin JG, Tsai CC, Chen TH, et al. Effects of percutaneous electrical stimulation on peripheral nerve regeneration using silicone rubber chambers. J Biomed Mater Res 2001;57:541-9.

21. Lu MC, Ho CY, Hsu SF, Lee HC, Lin JH, Yao CH, et al. Effects of electrical stimulation at different frequencies on regeneration of transected peripheral nerve. Neurorehabil Neural Repair
2008;22:367-73.

22. Rateb EE, Amin SN, El-Tablawy N, Rashed LA, El-Attar S. Effect of melatonin supplemented at the light or dark period on recovery of sciatic nerve injury in rats. EXCLI J 2017;16:138-50.

23. Kaya Y, Sarıkcığlu L, Aslan M, Kencebay C, Demir N, Derin $\mathrm{N}$, et al. Comparison of the beneficial effect of melatonin on recovery after cut and crush sciatic nerve injury: a combined study using functional, electrophysiological, biochemical, and electron microscopic analyses. Childs Nerv Syst 2013;29:389401.

24. Turgut M, Kaplan S. Effects of melatonin on peripheral nerve regeneration. Recent Pat Endocr Metab Immune Drug Discov 2011;5:100-8.

25. Lehman NL, Johnson LN. Toxic optic neuropathy after concomitant use of melatonin, zoloft, and a high-protein diet. J Neuroophthalmol 1999;19:232-4. 